Check for updates

The BMJ

Cite this as: $B M J 2021 ; 373: n 1112$ http://dx.doi.org/10.1136/bmj.n1112 Published: 28 April 2021

\section{Covid-19: One dose of vaccine cuts risk of passing on infection by as much as $50 \%$, research shows}

\author{
Elisabeth Mahase
}

Adults infected with covid-19 three weeks after receiving one dose of the Pfizer-BioNTech or Oxford-AstraZeneca vaccine were $38-49 \%$ less likely to pass the virus on to their household contacts than people who were unvaccinated, a preprint released by Public Health England has shown.

The research looked at the proportion of household contacts who tested positive 2-14 days after vaccinated index cases, comparing this with households where the index case was unvaccinated. The team said that protection was seen from around 14 days after vaccination, and similar levels were observed regardless of the age of cases or contacts.

Public Health England said that this protection was on top of the reduced risk of a vaccinated person developing symptomatic infection in the first place, which was around $60-65 \%$ four weeks after one dose of either vaccine.

“This is very promising," said Deborah Dunn-Walters, the British Society for Immunology's covid-19 taskforce chair and professor of immunology at the University of Surrey. "While this study brings welcome news, we must not be complacent... It is still very important for us all to get two doses of the covid-19 vaccine to ensure we receive the optimal and longest lasting protection, both for ourselves and our communities."

\section{Emerging evidence}

The preprint included people with at least 14 days of observable follow-up from the date of specimen collection from the index case (from 4 January to 28 February 2021; secondary cases up to 14 March 2021).

A total of 552984 residential households with 2-10 people where there was at least one case were included. In households where the index case was not vaccinated before testing positive, the study found 96898 secondary cases from 960765 household contacts (10.1\%).

Meanwhile, in households where the index case received the AstraZeneca vaccine 21 days or more before testing positive, 196 secondary cases were seen in 3424 contacts (5.72\%). With the Pfizer vaccine (one dose 21 days or more before testing positive), 371 secondary cases were found in 5939 contacts $(6.25 \%)$.

The researchers adjusted for age of index case and contact, sex of index case and contact, government office region, calendar week of index case, deprivation quintile of the local area, and household type. They said that the results "persisted after adjustment for measured covariates." However, they also noted limitations to their work, including that they could not identify asymptomatic cases.
"These findings provide emerging evidence that receipt of at least one dose of either vaccine reduces transmission of SARS-CoV-2 from a diagnosed case to other persons in the household setting," the preprint said. "It will be important to assess if there are any further reductions in transmissibility from receiving a second dose of vaccine when such data become available."

\section{Antibodies}

Public Health England's head of immunisation, Mary Ramsay, said, "Vaccines are vital in helping us return to a normal way of life. Not only do vaccines reduce the severity of illness and prevent hundreds of deaths every day, we now see they also have an additional impact on reducing the chance of passing covid-19 on to others. I encourage anyone who is offered a vaccine to take it as soon as possible."

The latest Office for National Statistics (ONS) estimates show that $59.8-70.6 \%$ of adults in the UK have received at least one dose of a covid-19 vaccine as of 16 April, while $19.5-23.3 \%$ of adults are fully vaccinated (two doses). ${ }^{2}$

ONS data also estimate that seven in 10 adults in England would have tested positive for antibodies against SARS-CoV-2 in a blood test in the week ending 11 April 2021, suggesting that they had the infection in the past or have been vaccinated. In Wales, Scotland, and Northern Ireland this figure was six in 10 adults.

\section{Harris RJ, Hall JA, Zaidi A, Andrews NJ, Dunbar JK, Dabrera G. Impact of vaccination on household transmission of SARS-COV-2 in England. 2021 [preprint]. https://khub.net/documents/135939561/390853656/Im- pact+of+vaccination+on+household+transmission+of+SARS-COV- 2+in+England.pdf/35bf4bb1-6ade-d3eb-a39e- 9c9b25a8122a?t=1619551571214. \\ 2 Office for National Statistics. Coronavirus (COVID-19) Infection survey, antibody and vaccination data for the UK: 28 Apr 2021. 28 Apr 2021. https://www.ons.gov.uk/peoplepopulationandcommunity/healthandsocial- care/conditionsanddiseases/articles/coronaviruscovid19infectionsurveyan- tibodydatafortheuk/28april2021\#behavioural-changes-following-vaccina- tion-in-the-uk.}

This article is made freely available for use in accordance with BMJ's website terms and conditions for the duration of the covid-19 pandemic or until otherwise determined by BMJ. You may use, download and print the article for any lawful, non-commercial purpose (including text and data mining) provided that all copyright notices and trade marks are retained. 